\title{
The constancy of the orientation of the visual field
}

\author{
HANS WALLACH and JOSHUA BACON \\ Swarthmore College, Swarthmore, Pennsylvania 19081
}

\begin{abstract}
Evidence is presented that the perceived immobility of the environment during tilting of the head from side to side results from a compensating process. This compensating process operates well only when peripheral vision is present. An objectively stationary environment was, for instance, not perceived as immobile during head tilting when vision was confined to the macular region of the retina. The compensating process could be rapidly altered by exposure to environmental tilting during and dependent on head tilting. Such adaptation had the result that some environmental tilting that normally is perceived led to apparent immobility.
\end{abstract}

Much effort has gone into the investigation of the fact that the visual environment is perceived as immobile when one turns or nods one's head. Such head movements cause displacements of the environment relative to the head that are not perceived; owing to a learned compensating process, which has been called "constancy of visual direction"' by us and "position constancy" by others. ${ }^{1}$ There is a third head movement that changes the relation between the visual environment and the head, a tilting of the head from side to side. This movement causes a change in the orientation of the environment relative to the head. This change in orientation is also not perceived. Is that, too, the result of a compensating process?

There is an alternative explanation for the perceived immobility of the visual environment during head tilting. This explanation would assume that all stimulation produced by changes of the orientation of the environment relative to the head is discounted, as long as these changes occur during a tilting movement. If that were the case, it would not matter whether these changes resulted only from head movements or were also caused by objective or optical tilting of the environment. If, on the other hand, perceived immobility of the environment during head tilting were limited to objective immobility and to some objective orientation changes, and if other objective tilting of the environment were correctly perceived, we would have evidence for the operation of a compensation process. It is obvious that our question can only be answered if we have the means to cause the visual environment of a subject to tilt actually or optically while he tilts his head from side to side.

An answer to this question is easily obtained when one wears a single left-right reversing prism in front of one eye while the other eye is covered. Normally, tilting of the head causes a change in alignment

This work was supported by Grant 11089 from the National Institute of Mental Health to Swarthmore College, Hans Wallach, principal investigator. between the head and the environment, and this change causes visual stimulation. This stimulation is best described by selecting the head as frame of reference for the change in alignment between head and environment. When this is done, a clockwise tilting of the head, for instance, can be said to be accompanied by a counterclockwise tilting of the environment relative to the head. ${ }^{2}$ This relative tilting of the environment is not perceived. When the reversing prism is worn and causes the counterclockwise relative environmental tilting to reach the eye as a clockwise tilting, one clearly perceives a clockwise tilting. This observation shows that we deal here with a compensating process that prevents the tilting of the environment relative to the head that is the counterpart of the head movement from being perceived, while at least one other tilting of the environment that occurs during a head tilting, the one seen through the prism, is correctly perceived.

The question, then, becomes: how accurate is this compensating process? How closely is the discounting of the stimulation produced by a relative environmental tilting limited to that tilting which is caused by the head's tilting movement? To answer this question, an apparatus is needed by which a tilting movement of the head can cause the tilting of a pattern in front of an observer, which represents his environment. The apparatus must also make it possible to vary the amount of this environmental tilting relative to the amount of the head tilting that causes it and to change the sense of the environmental tilting in relation to the sense of the head tilting. With the help of such an apparatus, it is possible to find out how much of an objective tilting can be added to or subtracted from the relative tilting that an immobile environment undergoes during head tilting, without this objective tilting being perceived. The smaller the amount of objective tilting that remains unnoticed, the more accurate is the compensating process.

The tilting of the head consists in a rotation of the head about an axis located at the level of the neck. 


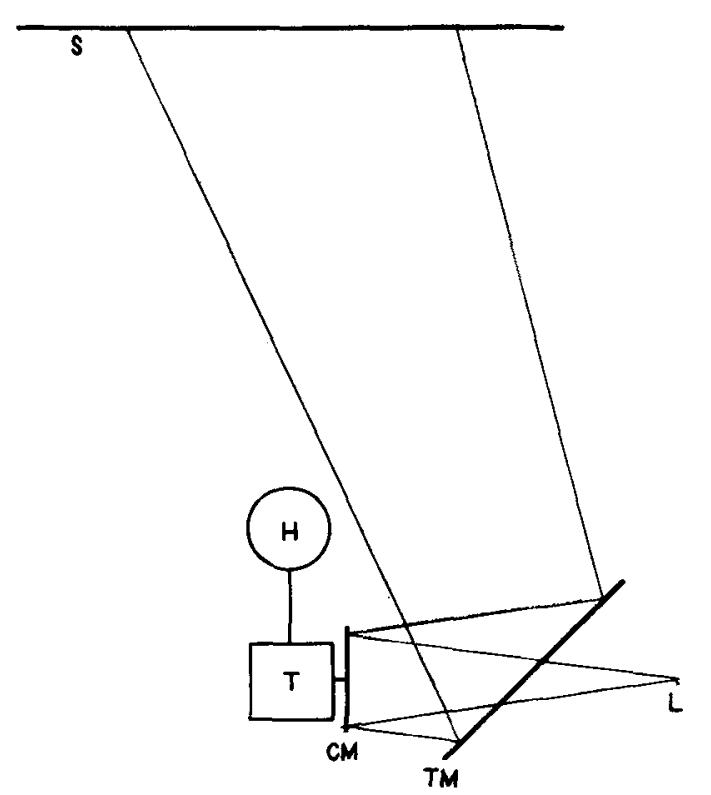

Figure 1. Our apparatus seen from above, drawn to scale. $H$ is the subjects head and $S$ the screen in front of him. $T$ is the variable ratio transmission. $\mathrm{CM}$ is the circular mirror that incorporates the radial pattern into the beam it reflects. The light that eventually reaches the screen starts from the light source $L$, passes through the transparent mirror TM, is reflected by $\mathrm{CM}$ and reflected again by TM. The limiting rays are drawn.

The relative tilting of the environment that results from it consists in a rotation about an axis that is the extension of the axis of the head rotation. The sense of this relative environmental rotation is, of course, opposite to the sense of the head rotation that causes it. Increasing or decreasing this relative environmental rotation is achieved by adding to the relative rotation produced by the head movement an objective rotation of the visual environment about the same axis, either in the sense opposite to or in the same sense as the head rotation. The amount of objective rotation so added without its being perceived as rotation characterizes the accuracy of the compensation process. Inasmuch as this objective rotation increases or decreases the relative environmental rotation proportionally, and since the latter results from the head rotation, the objective rotation must be proportional to the head rotation. To measure the accuracy of the compensation, the proportion of environmental rotation to the head rotation must be varied to map out the range of those proportions that do not lead to perception of environmental rotation. Our unit of measurement, then, is the orientation ratio (OR), the proportion of the environmental rotation to the head rotation, and the range of those orientation ratios that do not lead to perceived rotation of the environment, henceforth called the no-motion range, measures the accuracy of the compensation.

\section{Equipment}

The apparatus (see Figure 1) used to measure this no-motion range is based on a variable ratio transmission, a device that makes it possible to change continuously the ratio of the rate of rotation of the output shaft to that of the input shaft. The transmission was mounted in the back of the subject's chair, at the level of his neck, in such a way that its input shaft could be made to coincide with the axis of rotation of the tilting movements of his head. The output shaft, which in such transmissions forms a right angle with the input shaft, was horizontal. The back of a circular first-surface mirror, $20.5 \mathrm{~cm}$ in diameter, was so fixed to the end of the output shaft that the shaft was perpendicular to the plane of the mirror and coincided with its center. The mirror was covered with a concentric radial pattern of 2.5 -mm-wide paper strips. When this mirror was illuminated by a point scurce of light, the light reflected by the mirror would project a sharply outlined shadow of the radial pattern onto a screen. To avoid deformations of the projected pattern, the point source was located in the direction perpendicular to the center of the mirror. To bring the projected pattern onto a screen that was located in the subject's frontal plane $100 \mathrm{~cm}$ from his eyes, a transparent mirror was inserted between the light source and the circular mirror that projected the pattern, its center $51 \mathrm{~cm}$ from the input shaft of the transmission. Part of the light from the source would pass through the transparent mirror and be reflected by the circular mirror back to it. A portion of this reflected light that carried the projection of the radial pattern would be reflected by the transparent mirror onto the screen in front of the subject. The transparent mirror was so slanted that the center of the projected radial pattern was aligned with the input shaft of the transmission. This meant that when the output shaft turned the pattern on the circular mirror, the center of rotation of the projection of that pattern on the screen coincided with the input shaft and, hence, with the rotation center of the subject's tilting head movement. This particular arrangement caused the center of the radial pattern on the screen to coincide with the center of the relative rotation of the pattern that is due to head tilting. It thus provided proper superposition of the pattern rotation caused by the transmission on the relative rotation of the pattern that is the counterpart of the head tilting.

The alignment of the axis of the subject's head rotation with the fixed input shaft of the transmission was achieved in the following manner: The subject wore a headgear with a vertical bar attached in back that extended downward. On that bar rode an attachment that could be made to slide up and down and could be fastened in any desired location by an arresting screw. A coupling was fixed to this attachment, so that it extended horizontally backwards, perpendicularly to the subject's back. This coupling fitted over the input shaft of the transmission. ${ }^{3}$

After the headgear had been fastened on the subject's head, the experimenter positioned the coupling so that it underwent no lateral excursion when the subject tilted his head from side to side. Then the subject was seated in the apparatus on an adjustable chair and was raised until the coupling on his headgear was aligned with the input shaft and could be fitted over it.

The projection of the radial pattern on the screen in front of the subject subtended a visual angle of $40 \mathrm{deg}$. Depending on the setting of the variable transmission, this projection could be rotated in the sense with or against the head rotation, or it could remain stationary. The setting was changed by turning the control shaft with which a variable ratio transmission is equipped. A Veeder counter was connected to the control shaft; it enabled the experimenter to obtain instantaneously a reading of the setting, which, based on an initial calibration, informed him about the orientation ratio for which the transmission was set and in which sense the projected pattern turned, with or against the tilting of the subject's head.

During the procedure that measured the no-motion range, only the projected radial pattern was visible. Except for a $6-V$ headlight 
bulb tun on $4.5 \mathrm{~V}$ ac which served as the light source for the radial pattern, the room was dark. The light beam from this source was channeled through a tube, which restricted it to the circular mirror. Because of the two successive incidences of beam splitting, the intensity of light reaching the screen in front of the subject represented only a quarter of the output of the light source. The light reflected by the transparent mirror where the beam passed through it on the way to the circular mirror and the light that passed through the transparent mirror where it reflected the projected pattern onto the screen were intercepted by black screens.

\section{THE NO-MOTION RANGE}

Our experiment calls for determining the range of those rotations of the environment produced by our apparatus during head tilting that will not be perceived as rotations. This no-motion range, we have seen, measures the amounts of objective environmental rotation that can be added to or subtracted from the relative environmental rotation caused by the head rotation alone and remains unnoticed. The smaller the rates of unperceived environmental rotations, the more accurate is the compensation we are studying.

This no-motion range was determined through the following procedure:

The subject was seated in the apparatus and his head was attached to the transmission, as described above. He was asked to tilt his head steadily from side to side and report continuously the motions of the pattern in front of him. Having set the orientation ratio to an initial value that produced clearly perceived rotation of the pattern, the experimenter slowly lowered the rotation rate of the pattern until the subject no longer saw it move. At that point, the subject was asked to stop his head movements, and the experimenter read the transmission setting with a flashlight. For half the subjects, the initial pattern rotation was in the sense with the head tilting; gradually lowering the orientation ratio served to determine the limit of the nomotion range toward perceived rotation in that sense, the withlimit, for short. After that limit had been found, the experimenter set the orientation ratio for an initial value that produced clearly perceived pattern rotation in the sense against the tilting movements and gradually lowered the OR. When the subject stopped seeing that rotation, the transmission setting was read again. This reading represented the other limit of the no-motion range, the against-limit. For the other half of the 12 subjects who participated in this experiment, the two limits were obtained in the other order. The interval between the two limits on the orientation ratio scale was the width of the no-motion range.

We found that the mean width of the no-motion range amounted to .05 on the orientation ratio scale. To avoid having to deal with small fractions throughout this report we shall multiply all orientation ratios (OR) by 100 and call our units of measurement hOR. The mean limits of the no-motion range were 5.0 hOR for the with-limit and $.0 \mathrm{hOR}$ for the against-limit; .0 hOR is the condition where the pattern remains objectively stationary. The mean against-limit would normally be expected to involve objective orientation changes of the environment, that is, objective rotation of the pattern projection in the sense against the head tilting. That the mean against-limit coincided with environmental immobil- ity is a surprising finding. In the constancy of visual direction, the no-motion range straddles objective immobility; in fact, in the most recent study by Wallach and Canal (1976), the midpoint of the nomotion range always coincided with objective immobility. In our experiment, the mean width of the no-motion range reported above as .05 OR or 5 hOR was about twice as large as the mean width of the no-motion range reported for the constancy of visual direction.

Some casual observations prompted our next experiments. A few subjects had noticed some movement in the outer regions of the pattern when most of the pattern appeared to have become stationary. This suggested the possibility that the no-motion range is somewhat smaller for the peripheral visual field. We therefore report the no-motion range under two further conditions. In one of them, the circular mirror was covered with a black mask except for a small concentric circular region in the middle. This region projected a radial pattern on the screen that subtended a visual angle of $5 \mathrm{deg}$ and was thus restricted approximately to the macular regions of the subject's retinae. This condition will be called central display. In the condition that will be called peripheral display, a central region of the circular mirror was covered by a concentric circular mask that blocked out a central region of the projected radial pattern that subtended an angle of $10 \mathrm{deg}$. The projected pattern thus formed a ring $15 \mathrm{deg}$ of visual angle wide around a central dark region. A small fixation mark was projected on to the midpoint of the dark region, and, therefore, coincided with the invisible center of the radial pattern.

The mean no-motion ranges that were measured in connection with the adaptation experiments to be reported below were combined for each of the two conditions. The means we are here reporting are in each case the combined means of three experiments, with totals of 35 and 36 subjects contributing. In the case of the central display, the mean width of the no-motion range was large and amounted to 12.4 hOR. The corresponding mean for the peripheral display was 8.9 hOR, also high in comparison with the corresponding mean of the whole pattern display. Because the differences between the standard deviations of these means was greater than the differences between the means would warrantthe standard deviations were 8.3 and 5.2, respectively-we tested for a difference of the variances of the two means and found them significant $(F=2.60, p<.02)$. An even more striking difference becomes apparent when the limits of the two no-motion ranges are considered. Just as was the case with the full pattern display, neither one of the two no-motion ranges straddled objective immobility of the radial pattern. In the case of the central 
display, the mean limits of the no-motion range were 18.4 and 6.0 hOR, both in the with sense. The mean against-limit of the no-motion range, which had the with value of $6.0 \mathrm{hOR}$, was significantly different from zero at the .001 level $(t=5.7)$. In contrast, the mean limits of the no-motion range for the peripheral display were 9.0 and .1 hOR in the with sense. As in the case of the full pattern display, the againstlimit coincided with the point of objective immobility. By comparing the mean midpoints of the two nomotion ranges statistically, we found that the difference in the locations of the two no-motion ranges on the OR scale was highly significant $(p<.001$, $t=5.6)$.

To summarize our results: The compensation process we are investigating does not yield perceived immobility of the environment during head tilting when vision is restricted to the macular regions of the retinae. Under these conditions, the environment appears immobile during head tilting when it actually rotates in the sense with the head tilt at the average amount of 12 hOR. Since, on the other hand, the no-motion range obtained for the peripheral display comprises objective immobility, we conclude that the immobility of the environment ordinarily encountered is primarily based on peripheral vision.

\section{MODIFICATION OF THE COMPENSATION BY MEANS OF ADAPTATION}

It seems likely that the compensation whose nomotion range we have measured results from learning. ${ }^{4}$ This view would be confirmed if we could show that the compensation is rapidly altered by adaptation. We expected it to turn up a further difference concerning peripheral vs. central display. The following is a report on our demonstrations of adaptation in the constancy of environmental orientation (CEO), our short term for the compensation for environmental rotation caused by head tilting, on whose accuracy we have just reported. We obtained adaptive modification of CEO under two conditions, with the central display and with the peripheral display.

Adaptive modification of CEO is produced when a subject is exposed to an environment that, instead of being stationary, rotates during head tilting at a rate that depends on the rate of the tilting movement and stands in a constant ratio to it. The apparatus with which the no-motion range was measured provided such head-movement-dependent rotation of the subject's environment, namely, of the radial pattern display in front of him. We therefore made the exposure to the adaptation condition take place in that apparatus. The variable transmission was set to produce objective rotation of the display in the amount of $40 \mathrm{hOR}$, either in the sense with or against the head tilting. The subject was instructed to tilt his head continuously from side to side and watch the radial pattern in front of him. When exposure was to the peripheral display, he was asked to fixate the small mark in the center of the inner dark region.

Partial adaptation to environmental rotation during head tilting has two related manifestations. One of them is easily observed when, after the exposure period, the subject looks at the stationary display while tilting his head. When adaptation had been to rotation in the with sense, the subjects reported an apparent tilting of the test display in the sense against the head tilting. The other manifestation consists in a displacement of the no-motion range on the OR scale in the direction of the objective rotation to which the subject had been exposed. In other words, when the subject had partially adapted to an environmental rotation in the sense with the head tilting, a display that, to a lesser degree, turned in the sense with the head tilt would appear stationary, and the no-motion range after exposure would represent the objective conditions that then produced perceived environmental immobility. By comparing the midpoint of the no-motion range (nomotion point, for short) measured after exposure to the adaiptation conditions with the no-motion point gotten before exposure, a measurement of the adaptation effect can be obtained. Since adaptation means that the OR value of the no-motion point becomes higher, we deducted the OR value of the preexposure no-motion point from that of the postexposure no-motion point to measure the adaptation effect.

The procedure in all of our adaptation experiments was this: The preexposure no-motion range was measured. Then the apparatus was set to produce a 40 hOR rotation of the display, and the subject tilted his head from side to side for $10 \mathrm{~min}$. Immediately following this exposure period, the no-motion range was measured again. A different group of 12 subjects, paid undergraduates, participated in each of our six experiments.

\section{Basic Experiments}

In Experiments 1, 2, and 3, the same displays were used in the exposure and in the test condition. In Experiment 1, the subject adapted to the central display being rotated in the with sense and was tested with the central display also. Experiment 2 was identical except that adaptation was to the radial pattern turning in the against sense. In Experiment 3, the exposure was to the peripheral display turning in the with sense and the tests also employed the peripheral display.

Results. Significant adaptation effects were obtained under all three conditions (see Table 1). The effect, the difference between the post- and preexposure no-motion points, was 8.5 hOR for Experiment 1. It was smaller, namely, $5.9 \mathrm{hOR}$, in Experiment 2, 
where adaptation was in the against sense. ${ }^{5}$ This result, as well as the outcome of preliminary experiments, caused us to use rotation in the with sense in all our other experiments. The adaptation effect of 6.4 hOR obtained with the peripheral display (Experiment 3) was somewhat smaller than the effect obtained with the central display in Experiment 1. Although this difference was not quite significant ( $p<.05$ in a one-tailed test only), it ran parallel to the differences between the no-motion ranges reported earlier and suggested further exploration. The three experiments still to be reported provided the answer to the question of whether the difference in the adaptation effects obtained in Experiments 1 and 3 was due to the exposure or the test condition.

\section{Follow-up Experiments}

In Experiments 4 and 5, adaptation was to rotation of the whole display where the projection of the entire radial pattern, subtending a visual angle of $40 \mathrm{deg}$, was visible. (The first no-motion range reported above was obtained with that display.) In Experiment 4, the tests were done with the central display, and in Experiment 5, with the peripheral display. Nearly identical adaptation effects of 4.7 and $4.3 \mathrm{hOR}$, respectively, were obtained, indicating that the nature of the test display has little, if any, influence on the adaptation effect measured. Table 1 shows that adaptation to the whole display produced the smallest effects in all of our experiments. The difference between the effects of 8.5 and 4.7 that were obtained with exposure to the central display and to the whole display, respectively, with the test conditions identical, was significant at the .05 level $[t(22)=2.1]^{6}$

In Experiment 6, exposure was to the central display while testing was done with the peripheral display. The retinal regions involved in adaptation and tests were entirely different. A high adaptation effect of 7.3 hOR was obtained, indicating that the use of different displays in exposure and test in Experiments 4 and 5 was not responsible for the low effects obtained. The latter were due, rather, to the fact that adaptation was to the whole display. The high adaptation effect obtained in Experiment 6

Table 1

Mean Effect of 10-Min Exposure to Display Rotation of 40 hOR During Head Tilting Obtained in Six Experiments With Various Combinations of Exposure and Test Conditions, Expressed in hOR Units ( $=12$ Each)

\begin{tabular}{|c|c|c|c|c|}
\hline $\begin{array}{c}\text { Experiment } \\
\text { Number }\end{array}$ & $\begin{array}{c}\text { Di } \\
\text { Exposure }\end{array}$ & Test & $\begin{array}{c}\text { Adaptation } \\
\text { Effect }\end{array}$ & $t(11)$ \\
\hline 1 & Central & Central & 8.5 & 6.1 ** \\
\hline 2 & Central & Central & 5.9 & $8.3^{* *}$ \\
\hline 3 & Peripheral & Peripheral & 6.4 & $3.8 *$ \\
\hline 4 & Whole & Central & 4.7 & $7.3 * *$ \\
\hline 5 & Whole & Peripheral & 4.3 & $3.8 *$ \\
\hline 6 & Central & Peripheral & 7.3 & $5.7 * *$ \\
\hline
\end{tabular}

Table 2

Mean Increase of the Width of the No-Motion Range After Exposure and Mean Adaptation Effect on Near Limit Obtained in Six Experiments, Expressed in hOR Units

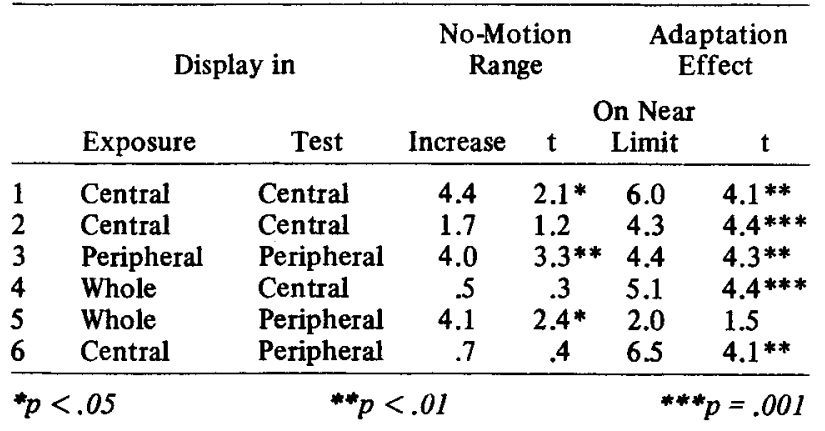

resulted from the fact that adaptation was to the central display.

We combined the results for Experiments 1 and 6, where exposure was to the central display in the with sense, and compared them with the combined results of those three experiments where exposure was either to the peripheral display (Experiment 3 ) or the whole display (Experiments 4 and 5). These latter displays involve peripheral regions of the retina in adaptation. The combined mean for the latter three experiments was 5.1 hOR, while the combined mean of the two experiments with adaptation to the central display was 7.9 hOR. $^{7}$ The two combined means were significantly different at the .02 level $[t(58)=2.5]$. When, on the other hand, the results of adaptation to rotation in the with sense were combined according to the displays used in the tests, the combined mean for Experiments 1 and 4, where the central display served in the tests, was $6.6 \mathrm{hOR}$, and the combined mean for Experiments 3, 5, and 6, where testing was done with the peripheral display, was 6.0 hOR. The nature of the test seems to have little effect on the measured effects.

These comparisons suggested to us that the adaptation effect was greater when, during the exposure period, vision was confined to the macula than when the periphery of the retina was involved, alone or together with the macula, and this is our tentative interpretation of the results. Another interpretation is, however, possible, namely, that the adaptation effect was greater the smaller the retinal area that was exposed to a rotating display. This alternative remains open, because the result of Experiment 3 was not significantly different from that of any of the other five experiments.

When partial adaptation in the constancy of visual direction was first measured by Wallach and Kravitz (1965), it was found that the width of the no-motion range was the same after adaptation as it was before the exposure period. ${ }^{8}$ That was not always the case in the experiments just reported. In three of the six adaptation experiments, there was a significant increase in the mean widths of the no-motion ranges 
after adaptation. An inspection of Table 2, where the mean increases in the no-motion ranges are listed, yields no association of increased no-motion width either with exposure or with test conditions. Neither is such an increase associated with large adaptation effects, which occurred in Experiments 1, 3, and 6. We have no explanation to offer for the three cases of expansion of the no-motion range after adaptation.

An important issue, however, is connected with such a widening of the no-motion range after adaptation. Such a widening could mean that adaptation, rather than shifting the whole no-motion range, merely altered one of its limits in the direction of adaptation. Such a one-sided expansion of the nomotion range would force us to change our views about the nature of adaptation. We would have to abandon the notion that in adaptation the normal compensation process that is measured in the preexposure test is superseded by another one that is in better agreement with the adaptation conditions. Instead, adaptation would make the compensation process less accurate so that, in addition to being to some extent in better agreement with the adaptation conditions, it also conforms to the normal viewing conditions. Such a one-sided expansion of the no-motion range would, of course, involve a shift of its midpoint and, since that is our indicator for an adaptation effect, would be accepted as an adaptation effect.

To find out whether such a one-sided expansion occurred in those of our experiments where adaptation was associated with an expansion of the nomotion range, we examined that limit of the postexposure no-motion range which was on the side toward the preexposure no-motion point. Did that near limit remain unaltered after adaptation or did it shift in the same direction as the no-motion point? The third column of Table 2 lists the adaptation effects as they manifest themselves in these near limits. In five of the six experiments, the differences between the post- and preexposure near limits were highly significant. Only in Experiment 5, where the smallest adaptation effect had been measured, was this difference so small that it failed to be significant. We can safely conclude that the kind of adaptation here investigated caused a shift in the no-motion range in the direction of the objective rotation to which the subject adapted and not merely an expansion of this range in that direction.

\section{SUMMARY}

We have shown that the perceived immobility of the visual environment during head tilting is due to a compensation process, by demonstrating that this immobility is confined to a limited range of rates of rotation of the environment relative to the head rotation that the tilting consists in. Outside this no- motion range, environmental rotations are correctly perceived. The mean no-motion range comprised objective rotations of the test pattern between the point of immobility and rotations with the head tilting amounting to $5 \%$ of the head rotation. Objective rotation in the same sense as the head rotation that exceeded $5 \%$ of the head rotation was correctly perceived, as were all objective rotations in the sense against the head tilting.

Similar results were obtained when the center of the test pattern was blotted out so that the visible pattern was on the retina only in extramacular location. When, however, the test pattern was confined to the macula, the mean no-motion range became much wider and ranged from a rotation of the test pattern of $6.0 \%$ of the head rotation to a rotation of $18.4 \%$ of the head rotation, both in the with sense. This means that an objectively stationary test pattern that is given within the macula appears to turn when the head is being tilted, in the sense against the head rotation. This was true of 31 of the 35 subjects who participated in this test. ${ }^{9}$

The underlying compensation process could be rapidly altered through adaptation, that is, by exposure to a condition where a pattern representing the visual environment rotated in a constant proportion of the head tilting. In the case of the exposure period of $10 \mathrm{~min}$ used by us, the adaptation effect was stronger when the rotating pattern was given only in the macula than when the periphery of the retina was also involved. This may be another indication that stimulation in the macula is processed differently from stimulation in the periphery where environmental orientation is concerned.

\section{REFERENCES}

Howard, I. P., \& Templeton, W. B. Human spatial orientation. London: Wiley, 1966.

Wallach, H., \& Canal, T. Two kinds of adaptation in the constancy of visual direction. Perception \& Psychophysics, 1976, 19, 445-449.

Wallach, H., \& Flaherty, E. W. Rapid adaptation to a prismatic distortion. Perception \& Psychophysics, 1976, 19, 261-266.

Wallach, H., \& Kravitz, J. H. The measurement of the constancy of visual direction and of its adaptation. Psychonomic Science, 1965, 2, 217-218.

Wallach, H., Yablick, G. S., \& Smith, A. Target distance and adaptation in distance perception in the constancy of visual direction. Perception \& Psychophysics, 1972, 12, 139-145.

\section{NOTES}

1. Representative articles on the constancy of visual direction are cited in Wallach, Yablick, and Smith (1972).

2. Some readers may find the term "relative to the head" vague and would prefer "retinally" in its place. We had to avoid the term "retinally" because the role of cyclotorsion in our experiments has, so far, remained unexplored. In referring to the proximal stimulation caused by head tilting, we have to be, for the time being, satisfied with "tilting relative to the head." 
3. We are grateful to Otto Hebel for constructing this apparatus.

4. For a hypothesis of how this learning comes about, see Wallach and Flaherty (1976).

5. This difference was significant only at the .05 level in a onetailed test.

6. All probabilities reported in this article are for two-tailed tests, unless otherwise stated.

7. An adaptation effect of 7.9 hOR amounts to $20 \%$ of total adaptation.

8. This was also the case in all subsequent work on adaptation in the constancy of visual direction in which the senior author participated, although that fact is usually not mentioned in the publications. There was, for instance, no increase in the mean postexposure no-motion ranges in the most recent experiments by Wallach and Canal (1976).

9. That a single vertical line appears to change its orientation when the subject moves his head from side to side has been observed before (see Howard \& Templeton, 1966, p. 193), but there appears to be no connection with our finding.

\section{(Received for publication December 17, 1975;} revision accepted February 14, 1976.) 